\title{
Common and Unique Neural Correlates of Autobiographical Memory and Theory of Mind
}

\author{
Jennifer S. Rabin ${ }^{1}$, Asaf Gilboa ${ }^{2}$, Donald T. Stuss ${ }^{3,4}$, Raymond A. Mar', \\ and R. Shayna Rosenbaum ${ }^{1,3}$
}

\begin{abstract}
There is an inconsistency regarding the relationship between thinking about personal past experiences during autobiographical memory (AM) and thinking about other people's mental states during theory of mind (ToM). Neuroimaging studies of $\mathrm{AM}$ and ToM consistently report overlap in the brain regions recruited. Lesion data, however, show that amnesic people with AM impairment can have intact ToM, suggesting that distinct neural mechanisms support these abilities [Rosenbaum, R. S., Stuss, D. T., Levine, B., \& Tulving, E. Theory of mind is independent of episodic memory. Science, 318, 1257, 2007]. The current fMRI study examined the functional and neural correlates of remembering one's own experiences in response to personal photos (AM condition) and imagining others' experiences in response to strangers' photos (ToM condition). AM and ToM
\end{abstract}

\section{INTRODUCTION}

Autobiographical episodic memory (AM) refers to detailed representations of past personally experienced events. Like AM, theory of mind (ToM) involves conscious awareness of mental states that are unobservable. In AM, the awareness is autonoetic (self-knowing) recollection of the past (Wheeler, Stuss, \& Tulving, 1997; Tulving, 1985); in ToM, it is inferring and imagining other people's current thoughts and feelings (Frith \& Frith, 2003; Premack \& Woodruff, 1978). Recent surveys of the disparate neuroimaging literatures on AM and ToM have noted that the two abilities resemble each other in the brain structures that they recruit (Buckner \& Carroll, 2007; Hassabis \& Maguire, 2007). The current fMRI study examines the basis for this resemblance, as well as how the two abilities might differ, by directly comparing AM and ToM within the same individuals.

A growing body of research suggests that the processes responsible for the retrieval of past personal episodes also serve non-mnemonic purposes. For example, fMRI studies

\footnotetext{
${ }^{1}$ York University, Toronto,Ontario, Canada, ${ }^{2}$ University of Haifa, Mount Carmel, Israel, ${ }^{3}$ Rotman Research Institute, Baycrest, Toronto, Ontario, Canada, ${ }^{4}$ University of Toronto, Toronto, Ontario, Canada
}

conditions were matched in terms of content and vividness, and were compared directly to a common baseline. Analyses revealed common activity within frontal and temporal-parietal regions, yet midline structures exhibited greater activity during AM. More specific analyses of event construction and detail elaboration revealed unique activation of the right hippocampus during AM construction, and of lateral regions, such as the right temporo-parietal junction (TPJ) during ToM elaboration. Moreover, a region of left hippocampus/perirhinal cortex appeared to be driven by event vividness. Thus, differences in AM and ToM emerge when a common baseline is used and temporal dynamics are taken into account. Furthermore, the right TPJ and related lateral regions, and not the hippocampus, may be needed for ToM, given that this ability is intact in amnesic people.

of AM and imagining scenes or future events consistently activate a nearly identical set of brain regions. These include the hippocampus and related medial temporal lobe structures, medial prefrontal cortex (mPFC), anterior cingulate cortex, posterior cingulate/retrosplenial cortex, precuneus, temporal poles (TP), and temporo-parietal junction (TPJ; Addis, Wong, \& Schacter, 2007; Szpunar, Watson, \& McDermott, 2007; Conway, Pleydell-Pearce, Whitecross, \& Sharpe, 2003; Okuda et al., 2003; for reviews, see Spreng, Mar, \& Kim, 2009; Buckner \& Carroll, 2007; Hassabis \& Maguire, 2007). The brain regions identified in studies of AM are also strikingly similar to those that emerge in separate neuroimaging studies of ToM (Saxe, Moran, Scholz, \& Gabrieli, 2006; Gallagher \& Frith, 2003), and the overlap between AM and ToM may be even greater than that observed between AM and future imagining (Spreng et al., 2009).

A number of theories have been offered to account for the neural correspondence observed across these diverse cognitive domains. One idea that borrows from the concept of autonoetic consciousness-awareness of the self as continuous through subjective time-is that a "capacity for self-projection" allows humans to mentally shift from the present moment into alternate times, places, and perspectives (Buckner, Andrews-Hanna, \& Schacter, 2008; Buckner \& Carroll, 2007). A complementary idea is 
captured by "constructive episodic-simulation hypothesis," which emphasizes that personal memories are not literal representations of the past, but instead, involve reassembling and binding together event details from earlier episodes. The constructive nature of memory also allows these details to be flexibly recombined to generate novel events (Schacter, Addis, \& Buckner, 2008; Schacter \& Addis, 2007). By contrast, building on the cognitive map theory (O'Keefe \& Nadel, 1978), Hassabis, Kumaran, Vann, and Maguire (2007) and Hassabis and Maguire (2007) view scene construction as central to different forms of mental displacement, including AM and ToM. Scene construction involves the retrieval and integration of relevant spatial information to provide a coherent context within which information can be imagined and manipulated.

Support for these theories as they relate to future imagining comes from studies of amnesic people with hippocampal damage who experience difficulties in recalling past personal events as well as in imagining events they have not yet experienced (Hassabis, Kumaran, Vann, et al., 2007; Rosenbaum et al., 2005; Klein \& Loftus, 2002; Tulving, 1985). However, AM impairment is not limited to narratives that involve the self and may also apply to the reconstruction of semantic narratives (well-known fairy tales and bible stories), which is unlikely to require the aspect of self-projection that relies upon personal experience and are low in spatial detail relative to other types of detail (Rosenbaum, Gilboa, Levine, Winocur, \& Moscovitch, 2009). Other research with amnesic people is at odds with these theories as they relate to ToM. For example, Rosenbaum, Stuss, Levine, and Tulving (2007) recently showed that two individuals with severely impaired AM due to damage that includes the hippocampus or its connections performed at the same level as controls on a large number of ToM tests. Furthermore, a behavioral study of healthy adults did not find a relationship between AM retrieval and ToM, although a relationship may exist in psychopathology (Corcoran \& Frith, 2003). This functional dissociation between AM and ToM demonstrates that the close overlap in brain regions needs to be more closely examined.

Evidence of a shared brain network underlying AM and ToM has been based on comparisons of independent neuroimaging studies investigating one ability or the other (e.g., Spreng et al., 2009; Buckner \& Carroll, 2007; Hassabis \& Maguire, 2007) or on direct comparison between a more laboratory-based list-learning measure of episodic memory retrieval and ToM (Andrews-Hanna, Saxe, Poulin, \& Buckner, 2007; see Gilboa, 2004 for a discussion of AM vs. laboratory-based episodic memory measures). To our knowledge, only one other study has directly compared AM to ToM, using the International Affective Picture System (IAPS; Lang, Bradley, \& Cuthbert, 2005) as cues, and found the same general pattern of shared activity between the two abilities as well as some patterns that were unique to each ability based on functional connectivity analysis (Spreng \& Grady, in press).
The current study also compared AM and ToM directly and against the same baseline measure to systematically examine areas common to both abilities, and areas of unique activity, taking into account certain processing demands believed to be shared by both. There is reason to believe that certain brain regions will be uniquely engaged, or play a predominant role, in one process over the other. For example, based on patient studies, the hippocampus may be specialized for representing details of personal experiences during AM (e.g., Rosenbaum et al., 2008) or in providing a spatial backdrop for those experiences to unfold (e.g., Hassabis \& Maguire, 2007). The TPJ, on the other hand, may play a specialized role in representing other people's mental states (Lombardo et al., in press; Saxe et al., 2006; Samson, Apperly, Chiavarino, \& Humphreys, 2004; Saxe \& Kanwisher, 2003) or in attentional reorienting (Cabeza, Ciaramelli, Olson, \& Moscovitch, 2008; Corbetta, Patel, \& Shulman, 2008; Mitchell, 2008). Alternatively, activity within these regions may be driven by the vividness of an event (Gilboa, Winocur, Grady, Hevenor, \& Moscovitch, 2004) or the richness and binding together of event details (Rosenbaum et al., 2009), whether the event is real or imagined, personal or impersonal.

To test these possibilities, the current fMRI study used a novel "family photos" paradigm based on one developed by Gilboa et al. (2004), in which participants recollected past events in response to personal photos (AM condition) and imagined ${ }^{1}$ possible events in response to photos belonging to unfamiliar people (ToM condition). The photos were matched in terms of perceptual, thematic, and temporal content, and provided a specific spatial context within which to create or recreate event details and mental states. The close similarity of the two conditions rendered any differences between AM and ToM all the more remarkable.

Recent fMRI studies of AM and imagining events suggest that neural activity associated with event memory and imagination may be influenced by the overall vividness of the generated event, and may further depend on whether activity is measured during the initial construction phase or elaboration phase of event generation (Addis et al., 2007; Gilboa et al., 2004). To address these factors, we took advantage of event-related fMRI by accounting for overall vividness and the amount of detail generated during event (re)construction. We also analyzed the time course of event generation by separately patterns of activity associated with the construction or search phase of an event and the elaboration phase when participants expand on event details, as collapsing across these phases can obscure important findings (Addis et al., 2007).

\section{METHODS \\ Participants}

Twenty healthy adults with normal or corrected-to-normal vision and no reported history of neurological or psychiatric 
illness participated in the study. Two participants were excluded due to scanner gradient artifact, leaving a total of 18 ( 9 men; mean age $=57.2$ years, $S D=8.0$, range $=$ 37-69; education $=16.39$ years, $S D=2.83$, range $=11-$ 21). Participants were right-handed, with the exception of one person who was left-handed. All participants gave informed written consent in accordance with the ethics committees at York University, Baycrest, and Sunnybrook Health Sciences Centre. Participants received monetary compensation for their time.

\section{Stimuli}

A unique, real-world "family photos" test of mental state attributions, used originally to examine AM in healthy adults (Gilboa et al., 2004), was adapted to create a separate but closely matched ToM condition and a low-level baseline condition to which both tasks were compared. Three conditions were developed for presentation to participants during scanning: recent $\mathrm{AM}$, remote $\mathrm{AM}$, and ToM. The AM condition consisted of 10 to 15 personal family photos of events that took place within the past 2 to 5 years (recent AM) or at least 15 years ago (remote $\mathrm{AM}$; these data are not presented in the current article). A relative or close friend of each participant collected the photos to reduce the likelihood that the events in the photos were mentally rehearsed prior to scanning. Relatives were instructed to find photos that included the participant to ensure consistency of self-related processing and to verify participants' presence at the events. Only photos depicting specific events (as opposed to portraits) were selected.

The ToM stimuli consisted of photos from people unknown to the participant that were matched to each recent $\mathrm{AM}$ photo according to event type (e.g., birthday party, picnic), scenery (e.g., indoor vs. outdoor), time period from which the photo was taken, and number of people present. Therefore, an equal number of recent AM and ToM photos were presented to each participant. The stimuli for the baseline condition consisted of scrambled photos. Matlab was used to scramble the pixels of each AM and ToM photo to produce a corresponding image matched in visual complexity and luminance. All photos were resized and gray-scaled.

E-Prime software (Psychology Software Tools, Pittsburgh, PA) was used to present the photos and instructions to participants in the scanner. Instructions were printed in black text on a white background. E-Prime was also used for the collection of reaction times and response data. Responses were made on an MR-compatible, five-button response box.

\section{Scanning}

Immediately prior to the scan, a short training session was provided to ensure that participants understood the instructions. The photos used in the training session were not used during the scan. At the beginning of each run, participants viewed a set of instructions that corresponded to one of the three conditions (i.e., recent AM, remote AM, or ToM). Each run contained five photos from one of the three conditions, which helped to maximize randomization. Each photo was presented for $20 \mathrm{sec}$. The order of conditions was pseudorandomized across the entire scanning session. The number of runs ranged from 6 to 9 (median $=8.5$ runs) and was determined by the number of recent and remote AM photos provided by participant (minimum of 10 photos per condition). Each run lasted $4 \mathrm{~min}$ and $10 \mathrm{sec}$.

For the recent AM and remote AM conditions, participants were presented with their own photos and asked to mentally recollect the event in each photo in as much detail as possible. They were told to focus on what they were thinking and feeling at the time. For the ToM condition, participants were presented with strangers' photos and asked to mentally generate a novel event/scenario for each photo. They were instructed to focus on what the people in the photo might have been thinking and feeling.

For each photo, participants were asked to press a button signaling the end of an initial construction phase (i.e., once an event was in mind) and the beginning of an elaboration phase (i.e., expanding on event details; Addis et al., 2007). The elaboration phase continued until the photo was no longer present on the screen. All photos remained on the screen for $20 \mathrm{sec}$, regardless of when responses were made, to maximize the recollection/ generation of details associated with each event.

Following each AM photo, participants were presented with the words "remember/know" to assess underlying recollection and familiarity processes (Gardiner, Ramponi, \& Richardson-Klavehn, 1998; Tulving, 1985). Participants were instructed to select "remember" (with a button press) if the event was specific to a time and place and they could re-experience it (i.e., they could mentally place themselves back in time to the event). They were instructed to make a "know" response if the event was familiar to them, but they could not recall any specific contextual or other experiential details associated with the event. Following each ToM photo, participants were presented with the words "detailed/vague" and were instructed to select "detailed" if their generated event was vividly imagined and to select "vague" if the event lacked specific details and was vague or unclear.

\section{Baseline Task}

The baseline task consisted of a brightness judgment in response to viewing scrambled photos. Scrambled photos were presented after each corresponding AM and ToM photo for $10 \mathrm{sec}$. Following each scrambled photo, participants were asked to indicate with a button press whether the photo was dark or light in brightness. 


\section{Postscan Interview}

Immediately following the scan, participants took part in an interview in which they viewed the same photos that had been presented in the scanner. Participants were asked to think back to the events they generated in the scanner, and to rate each AM and ToM event on a 7-point vividness scale ( $0=$ vague, $6=$ highly vivid). Participants also made a second "remember" versus "know" judgment for each AM event and a "detailed" versus "vague" judgment for each ToM event. To help prevent re-encoding or repeated retrieval of events in the scanner, participants were not made aware of the postscan interview until after the scanning session. The photos with the highest vividness ratings (approximately half of all photos) were selected for a semistructured interview, in which participants described the events as they had been recollected or imagined during the scan. The events were recorded and then transcribed for scoring. Scoring of the narratives was based on the autobiographical interview procedure described by Levine, Svoboda, Hay, Winocur, and Moscovitch (2002). Narratives were segmented into distinct details, which were further classified as internal (including event-specific, temporal, perceptual, spatial, and thought/emotion details) or external (including semantic and generic, nonspecific details).

Analyses of the postscan narratives were conducted by a trained rater who achieved high interrater reliability on the autobiographical interview (intraclass correlation coefficient, two-way random effects model; Shrout \& Fleiss, 1979) using a standard set of previously scored memories (Levine et al., 2002). A second rater scored a random sample of the narratives, and discrepancies (which were few and minor) were resolved by discussion. Coefficients for internal and external detail composites were .88 and .96 , respectively, for recall, and .89 and .94 for specific probe. Agreement on ratings composites was high for recall $(.79)$.

\section{Data Acquisition}

fMRI scanning was carried out at Sunnybrook Health Sciences Centre on a 3-T Signa MR scanner (Eclipse platform, 3T/94 quadrature head coil, VH3M4 software; GE Healthcare, Waukesha, WI). High-resolution, T1weighted images (3-D fast SPGR, TR $=7.2 \mathrm{msec}$, TE = $3.1 \mathrm{msec}$, IR-prepared TI $=300 \mathrm{msec}$, flip angle $=15^{\circ}$, matrix $=256 \times 192, \mathrm{FoV}=22 \mathrm{~cm} \times 16.5 \mathrm{~cm}, 124$ axial slices $1.4 \mathrm{~mm}$ thick) were acquired for structural reference. For blood oxygenation level dependent (BOLD) fMRI, T2*-weighted functional images were acquired using a spiral-in/out pulse sequence ( TR $=2000 \mathrm{msec}$, $\mathrm{TE}=30 \mathrm{msec}$, flip angle $=70^{\circ}$, matrix $64 \times 64$, FoV $=$ $20 \mathrm{~cm} \times 20 \mathrm{~cm}, 26$ axial slices $5 \mathrm{~mm}$ thick). Stimuli were presented using Avotec goggles; vision was corrected when necessary.

\section{Data Processing}

The event-related fMRI data were processed and analyzed using AFNI (Cox, 1996). The initial 10 time points of each run, in which transient signal changes occur as brain magnetization reaches a steady state, were excluded. Each participant's functional images were concatenated across runs and spatially realigned to one image (typically the 15 th volume of the first functional run) to reduce motion artifact. The peak range of head motion was less than $1.5 \mathrm{~mm}$ for all participants. Cardio and respiration data were used for physiological motion correction. Slice timing correction was performed using a Fourier interpolation. The realigned images were spatially normalized to a standard brain atlas space (Talairach \& Tournoux, 1988) and smoothed with a Gaussian filter of $6 \mathrm{~mm}$ full width at half-maximum. Functional data were overlaid on the average of all participants' anatomical scans.

For all participants, events were modeled with a gamma hemodynamic response function (HRF) that was applied when each photo appeared onscreen. Response times indicating the end of the construction phase and the beginning of the elaboration phase were available for only half of the participants due to an error in recording. For participants with these data, additional analyses examining neural activity during event construction and elaboration were conducted. For the construction phase, the HRF was applied when each photo appeared onscreen and ended when the elaboration phase began. The HRF for the elaboration phase was applied $1 \mathrm{sec}$ before the response time for each photo, given that neural changes associated with the retrieval of an AM are believed to occur approximately 1 sec before a manual response is made (Addis et al., 2007; Conway, Pleydell-Pearce, \& Whitecross, 2001). If a response was not made for a particular photo, that photo was excluded from the analyses.

Only the AM and ToM events rated as most vivid by participants in the postscan interview (i.e., vividness rating of 4 or greater) were included in the analyses (unless otherwise specified), as vividness is known to influence activity in regions associated with AM and episodic imagining (Gilboa et al., 2004). The postscan ratings were consistent with the within-scanner ratings of remember/know for the AM condition (Fisher's exact two-tailed test, $p<$ .001) and detailed/vague for the ToM condition (Fisher's exact two-tailed test, $p<.001)$. Moreover, ToM events for which participants reported drawing on their own past experiences were excluded to help ensure that ToM events were novel (see Results). For the baseline condition, brightness judgments were typically made within the first $4 \mathrm{sec}$ of photo onset. Thus, the last $6 \mathrm{sec}$ were excluded from analysis.

\section{Statistical Analysis Approach}

Group analyses consisted of a voxelwise, mixed-model ANOVA, with participants as a random factor and conditions 
as a fixed factor. To identify regions commonly engaged by AM and ToM, we compared the BOLD signal associated with AM and ToM to a common baseline for each participant and then entered these contrasts into a conjunction analysis. To determine regions differentially engaged by AM and ToM events, a direct comparison between AM and ToM was computed for each participant and then entered into a random effects one-sample $t$ test.

As previously mentioned, construction and elaboration data were available for only half of the participants due to a recording error. Using these data, we further compared the BOLD signal associated with AM and ToM event construction and elaboration to a common baseline, such that the following contrasts were computed for each participant: (a) AM construction versus baseline, (b) ToM construction versus baseline, (c) AM elaboration versus baseline, and (d) ToM elaboration versus baseline. These contrasts were then used to compute two conjunction analyses: (a) AM construction and ToM construction; and (b) AM elaboration and ToM elaboration. To determine regions differentially engaged, a direct comparison between AM construction and ToM construction was computed for each participant and then entered into a random effects onesample $t$ test. The same analysis was computed for AM and ToM elaboration.

To determine if the high vividness ratings associated with AM events (see Results), and not AM itself, was driving the more extensive pattern of activity observed during AM relative to ToM, we compared the BOLD signal associated with ToM events participants rated as most vivid (vividness rating of 6) with the BOLD signal associated with ToM events classified as vague/unclear (vividness rating between 0.5 and 2.5). Vivid ToM and vague ToM events (relative to baseline) were extracted from participants and then entered into a random effects two-sample $t$ test. ${ }^{2}$ The logic here is that if vividness is driving the activity of the core network, then we would expect the core network to exhibit greater activity during the generation of vivid ToM events compared to ToM events rated as vague or unclear. A similar analysis of vividness within the AM condition was not possible due to the low number of vaguely recollected personal events (vividness rating between 0.5 and 2.5).

Statistically significant brain activity was established using a voxel-cluster threshold technique, $p<.005$, corrected, minimum cluster size $120 \mathrm{~mm}^{3}$, and having a connectivity radius of $2 \mathrm{~mm}$ (i.e., two clusters need to be separated by at least $2 \mathrm{~mm}$ to be classified as different). A threshold of $p<.01$, corrected, was applied to contrasts that were divided into construction and elaboration phases and to the comparison of vivid and vague ToM events, as these involved fewer participants (see Results). The coordinates of clusters reported correspond to the peak $t$ value. Because of our a priori hypothesis regarding the role of the hippocampus and surrounding structures in AM and/or vividness, we applied a more liberal threshold of $p<.05$, corrected, when examining activity in this region.

\section{RESULTS}

The majority of analyses included only events rated as vivid by participants (see above). Therefore, $28 \mathrm{AM}$ photos from 10 participants and 64 ToM photos from 15 participants rated as vague/unclear (i.e., vividness rating less than 4) were excluded. An additional five ToM events for which a participant reportedly drew on past experiences and another five ToM events affected by scanner gradient artifact in a separate participant were excluded. Therefore, 11.9\% of AM and 31.5\% of ToM photos were excluded from analyses. This resulted in each participant contributing an average of $11 \mathrm{AM}$ events $(S D=3.4$, range $=6-15)$ and 8.9 ToM events $(S D=4.5$, range $=1-15)$ to the analyses. Moreover, vivid AM and ToM events had an average rating of $5.61(S D=0.37)$ and $4.73(S D=0.62)$, respectively. With respect to the baseline condition, each participant contributed an average of 38.33 scrambled photos to the analyses. A nonparametric Wilcoxon signed-rank test indicated that AM events were significantly more vivid than ToM events $(Z=3.29, p<.05)$. To address this discrepancy, we conducted a separate analysis contrasting ToM events that were highly vivid with those that were vague to determine if a general vividness effect accounts for the pattern of activity observed in the AM condition (see below). Analyses of the postscan narratives indicated that the number of internal details generated in response to AM and ToM photos did not differ significantly (Wilcoxon signed-rank test, $Z=$ $-1.95, n s){ }^{3}$ A Friedman test indicated a significant difference in detail type within each condition [AM: $\chi_{r}{ }^{2}=36.28$ $(4, n=14), p<.001$; ToM: $\chi_{r}{ }^{2}=42.27$ ( $\left.4, n=14\right), p<$ .001]. Post hoc multiple comparisons (Sheldon, Fillyaw, \& Thompson, 1996) indicated that within the AM condition, participants produced significantly more event-specific details compared to temporal, perceptual, and thought/ emotion details. There was also a trend toward participants producing significantly more event-specific details relative to place details. In the ToM condition, participants produced significantly more event-specific details relative to place, temporal, and perceptual details, and significantly more emotion/thought details compared to temporal details. ${ }^{4}$

\section{Regions Commonly Engaged by Vivid AM and ToM Events}

Analyses revealed activity in a set of brain regions similar to the ones that have been reliably activated in previous studies of AM and ToM, among other abilities (Spreng et al., 2009; Buckner \& Carroll, 2007; Hassabis \& Maguire, 2007), including recent studies directly comparing the two (Spreng \& Grady, in press) or directly comparing a laboratory-based episodic memory test to a false-belief test of ToM (Andrews-Hanna et al., 2007). These regions include bilateral hippocampus and perirhinal cortex (BAs 35 and 36), bilateral paracingulate cortex (BA 32), bilateral frontal poles (BA 10), left ventrolateral PFC (VLPFC; BA 47), bilateral posterior cingulate/retrosplenial cortex 
(BA 29/30), bilateral middle temporal gyrus (MTG; BA 21), right TP (BA 38), and left middle occipital cortex (BA 19; see Table 1).

\section{Regions Differentially Engaged by Vivid AM and ToM Events}

Having recreated the core network of brain regions known to be shared between AM and ToM, we contrasted the two conditions to identify regions of differential and unique activity. Analyses revealed greater activity within bilateral perirhinal cortex (BAs 35 and 36), mPFC, and midline regions during AM relative to ToM. Moreover, a conjunction analysis of AM and ToM indicated that activity in bilateral thalamus (medial dorsal nucleus) was unique to AM, whereas the direct comparison indicated that activity in the right middle frontal gyrus (BA 8), left VLPFC (BA 45), left precentral gyrus (BA 4), and left supramarginal gyrus/angular gyrus (BA39) was unique to ToM (see Table 1; Figure 1).

\section{Regions Commonly Engaged by Vivid AM and ToM Event Construction and Elaboration}

Subsequent comparisons of the construction and elaboration phases of vividly generated AM and ToM events were computed. Two AM and four ToM events from three participants were excluded from analyses, as reaction time data were unavailable for these events. Therefore, nine participants contributed an average of 10.67 vivid AM events $(S D=3.39$, range $=8-15)$ with an average rating of $5.75(S D=0.21)$ and 8.67 vivid ToM events $(S D=4.56$, range $=1-15)$ with an average rating of $4.96(S D=0.71)$. Nonparametric Wilcoxon signed-rank tests indicated a significant difference between AM and ToM postscan vividness ratings $(Z=-2.10, p<.05)$ and that AM event construction took significantly less time $(M=3.80 \mathrm{sec}$, $S D=1.32 \mathrm{sec}$, range $=1.11-11.52 \mathrm{sec})$ than ToM event construction $(M=5.79 \mathrm{sec}, S D=2.73 \mathrm{sec}$, range $=1.28$ $14.56 \mathrm{sec} ; Z=-2.07, p<.05)$. Event elaboration began after the construction phase ended and lasted the remaining $20 \mathrm{sec}$ (Figure 2).

Regions of common activity during AM and ToM construction included right entorhinal/perirhinal cortex (BA 28/ 35), left parahippocampal cortex (BA 36), bilateral paracingulate cortex (BA 32), bilateral frontal poles (BA 10), left VLPFC (BA 47), bilateral posterior cingulate cortex/ precuneus (BA 31/23), and bilateral inferior occipital cortex (BA 18/19; see Table 2).

As in the construction phase, regions of common activity during $\mathrm{AM}$ and ToM elaboration included right perirhinal (BA 35), right entorhinal (BA 28), and left entorhinal/ perirhinal cortex (BA 28/35); bilateral paracingulate cortex (BA 32); left frontal pole (BA 10); bilateral posterior cingulate cortex/precuneus (BA 31/23/30), although extending here into retrosplenial cortex; left VLPFC (BA 47); and right inferior occipital cortex (BA 18/19), although to a lesser extent. However, unlike AM and ToM construction, AM and ToM elaboration both recruited bilateral anterior cingulate cortex (BA 25), left dorsomedial PFC (BA 9), bilateral hippocampus, left MTG (BA 21), and left TP (BA 38; see Table 2).

\section{Regions Differentially Engaged by Vivid AM and ToM Event Construction and Elaboration}

Conjunction analysis of AM and ToM event construction versus baseline indicated that $\mathrm{AM}$ construction uniquely engaged bilateral hippocampus, extending into parahippocampal cortex. A direct comparison between AM and ToM construction revealed greater activity in right entorhinal/ perirhinal cortex (BA 28/35), left entorhinal and parahippocampal cortices (BAs 34 and 36/37), right superior frontal gyrus (BA 8), right dorsolateral PFC (BA 9), and bilateral lingual gyrus (BA 18/19) during AM (Table 2). Conversely, no regions were engaged to a greater extent during ToM event construction.

During the elaboration phase, AM was again associated with greater activity in mostly medial regions, including bilateral medial-superior PFC (BAs 9, 10, and 46), left paracingulate cortex (BA 32), bilateral perirhinal cortex (BAs 35 and 36), bilateral posterior cingulate/retrosplenial cortex (BA 29/30/23), and bilateral precuneus (BA 18/31). Based on this analysis, the bilateral hippocampus was engaged to the same extent in both conditions. In contrast, ToM uniquely engaged lateral regions, including left VLPFC (BA 44/45), left precentral gyrus (BA 4), left VLPFC/superior temporal gyrus (STG; BA 44/22), left STG (BA 22), right TPJ (BA 39), and left supramarginal gyrus (BA 40; see Table 2).

\section{Regions Associated with Event Vividness}

To determine whether vividness, which was rated as significantly greater for AM than ToM events, accounts for the more extensive pattern of activity during AM, we compared ToM events rated as most vivid $(M=6.0, S D=0)$ with those that were classified as vague or unclear $(M=1.77$, $S D=0.29$, range $=0.5-2.5$ ). A nonparametric Wilcoxon signed-rank test confirmed that vivid versus vague ratings differed significantly $(Z=-2.71, p<.05)$. Vivid ToM events and vague ToM events (relative to baseline) were extracted from nine participants (only a subset of participants was included in this analysis in order to closely match the total number of vivid and vague ToM events). Therefore, each participant contributed an average of 5.11 vivid ToM events $(S D=4.6$, range $=1-15)$ and 3.44 vague ToM events $(S D=2.3$, range $=1-7)$ to the analysis.

Compared to vaguely imagined ToM events, vivid ToM events were associated with greater activity in a region of left perirhinal cortex (BA 35) extending into the left 
Table 1. Regions Commonly and Differentially Engaged by Vivid AM and ToM Events

\begin{tabular}{|c|c|c|c|c|c|}
\hline \multirow[b]{2}{*}{ Brain Regions } & \multicolumn{3}{|c|}{ Coordinates } & \multirow[b]{2}{*}{$t$} & \multirow[b]{2}{*}{ Vol. } \\
\hline & $x$ & $y$ & $z$ & & \\
\hline \multicolumn{6}{|l|}{$A M$ and $T O M>$ Baseline } \\
\hline \multirow[t]{2}{*}{ R. Hippocampus/perirhinal cortex (BA 35) } & 24 & -21 & -15 & 5.37 & 392 \\
\hline & 29 & -27 & -9 & 5.02 & 184 \\
\hline \multirow[t]{2}{*}{ L. Hippocampus } & -24 & -13 & -21 & 3.22 & 543 \\
\hline & -30 & -9 & -19 & 3.94 & 179 \\
\hline R. Perirhinal cortex (BA 35) & 29 & -28 & -19 & 5.37 & 402 \\
\hline R. Parahippocampal cortex (BA 27) & 13 & -32 & -4 & 7.39 & 162 \\
\hline L. Perirhinal cortex (BA 36) & -23 & -35 & -16 & 5.37 & 24075 \\
\hline L. Paracingulate cortex (BA 32) ${ }^{a}$ & -2 & 46 & 2 & 7.39 & 269 \\
\hline R. ACC (BA 25) ${ }^{\mathrm{a}}$ & 2 & 14 & -3 & 4.28 & 1122 \\
\hline L. Frontal pole (BA 10) ${ }^{\mathrm{a}}$ & -6 & 53 & 1 & 5.37 & 2594 \\
\hline R. Medial frontal gyrus (BA 9) & 6 & 51 & 23 & 5.37 & 3684 \\
\hline L. Ventrolateral PFC (BA 47) & -43 & 29 & -2 & 5.37 & 461 \\
\hline L. Dorsolateral PFC (BA 9) & -53 & 11 & 26 & 4.28 & 270 \\
\hline \multirow[t]{2}{*}{ L. PCC/RSC (BA 29/30) ${ }^{a}$} & -2 & -56 & 11 & 7.39 & 2987 \\
\hline & -3 & -56 & 11 & 6.54 & 3565 \\
\hline L. Middle temporal gyrus (BA 21) & -56 & -8 & -14 & 5.37 & 476 \\
\hline R. Middle temporal gyrus (BA 21) & 57 & & -18 & 5.37 & 346 \\
\hline R. Temporal pole (BA 38) & 44 & & -26 & 3.94 & 249 \\
\hline R. Fusiform gyrus (BA 19) & 44 & -73 & -11 & 6.54 & 372 \\
\hline L. Middle occipital cortex (BA 19) & -48 & -71 & -8 & 6.54 & 1284 \\
\hline \multicolumn{6}{|l|}{$A M>T O M$} \\
\hline R. Perirhinal cortex (BA 35)* & & -25 & -11 & 2.11 & 218 \\
\hline L. Perirhinal cortex (BA 36)* & -31 & -28 & -23 & 2.11 & 1235 \\
\hline R. Frontal pole (BA 10) ${ }^{\mathrm{a}}$ & 2 & 51 & 7 & 4.28 & 661 \\
\hline R. Frontal pole (BA 10) & 2 & 64 & 21 & 3.21 & 487 \\
\hline L. Paracingulate cortex (BA 9/32) & -1 & 44 & 29 & 3.94 & 843 \\
\hline L. PCC/RSC (BA 23/29/30) a & -1 & -55 & 13 & 4.28 & 6078 \\
\hline R. Thalamus (medial dorsal nucleus) ${ }^{\mathrm{a}}$ & 3 & -14 & 7 & 5.02 & 1021 \\
\hline L. Middle occipital cortex (BA 18/19) & -39 & -84 & 16 & 3.03 & 169 \\
\hline R. Superior occipital cortex (BA 39/19) & 36 & -71 & 25 & 3.94 & 423 \\
\hline \multicolumn{6}{|l|}{$T o M>A M$} \\
\hline R. Middle frontal gyrus (BA 8) & 43 & 20 & 44 & 3.22 & 233 \\
\hline L. Ventrolateral PFC (BA 45) & -49 & 34 & 3 & 3.94 & 209 \\
\hline L. Precentral gyrus (BA 4) & -50 & -10 & 45 & 3.22 & 167 \\
\hline L. Supramarginal/angular gyrus (BA 39) & -37 & -51 & 30 & 3.94 & 291 \\
\hline
\end{tabular}

All activations reported survived a threshold of $p<.005$ (corrected). For each cluster of activation, the Talairach coordinates of the maximally activated voxel within each structure are reported, as indicated by the highest $t$ value. $\mathrm{AM}=$ autobiographical memory; ToM $=$ theory of mind; Vol. $=$ cluster volume $\left(\mathrm{mm}^{3}\right) ; \mathrm{R}=$ right; $\mathrm{L}=\mathrm{left} ; \mathrm{BA}=\mathrm{Brodmann}$ 's area; $\mathrm{ACC}=$ anterior cingulate cortex; PFC $=$ prefrontal cortex; PCC = posterior cingulate cortex; RSC $=$ retrosplenial cortex.

In the case where two coordinates are provided due to slight differences in the peak area of activation, the first corresponds to AM and the second to ToM.

${ }^{a}$ Although the only local maximum for this structure was lateralized, this activation was bilateral.

*A priori region of interest, $p<.05$. 


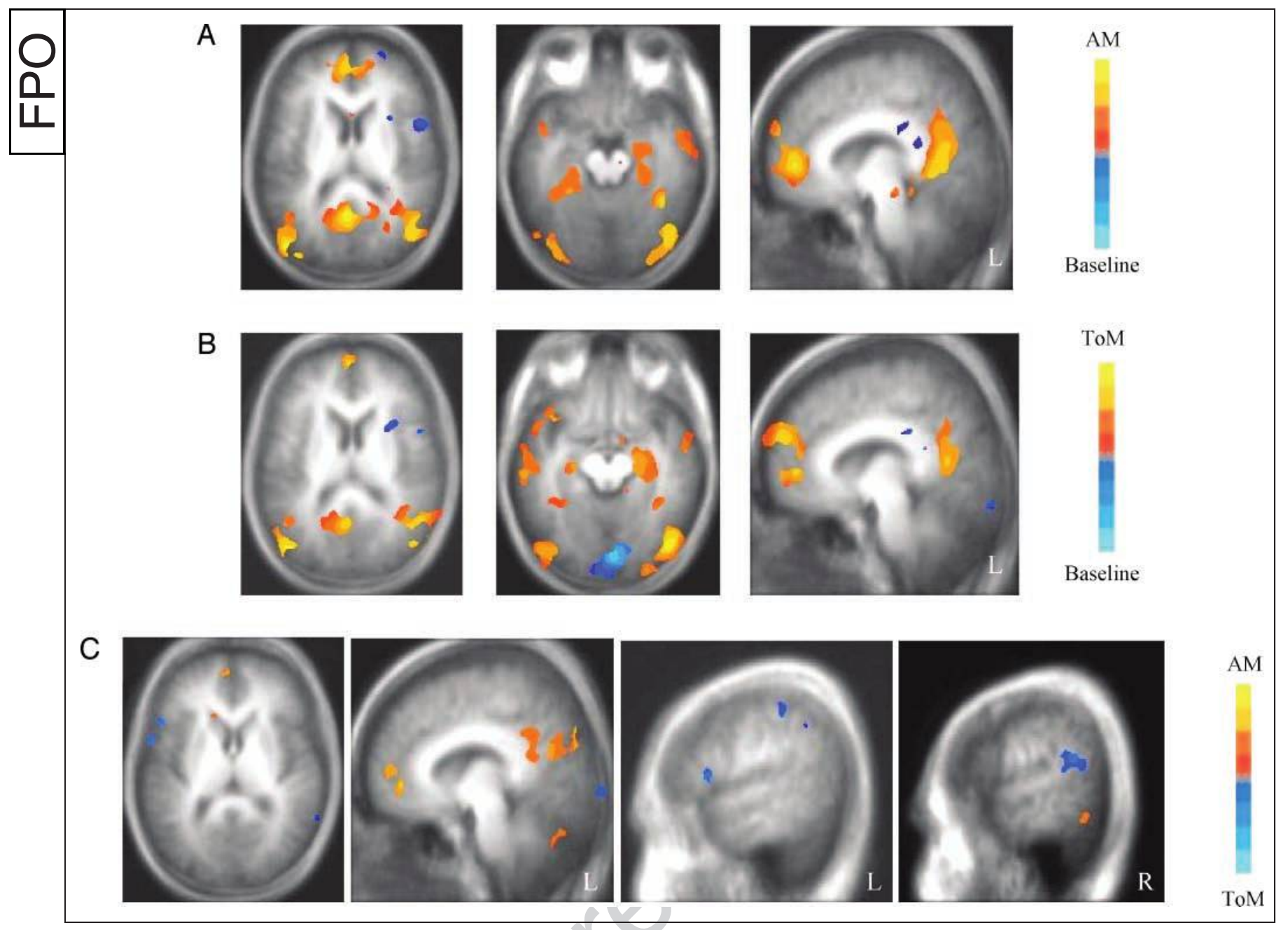

Figure 1. (A and B) Axial and sagittal slices of the left hemisphere illustrating common brain regions activated during AM and ToM detail elaboration relative to baseline, respectively. (C) Axial and sagittal slices of the left hemisphere and a sagittal slice of the right hemisphere (far right) illustrating regions differentially engaged during AM and ToM detail elaboration (for coordinates of these and other regions not displayed, see Table 2). All images are at a threshold of $p<.01$ (corrected), except for the ToM image depicting hippocampal activation (middle image, middle row), which is at a threshold of $p<.05$ (corrected). The functional maps are overlaid on the average anatomical image from all participants. Activations shown include areas $>120 \mathrm{~mm}^{3}$. Images follow neurological convention (left side of the brain is presented on the left)

hippocampus, right perirhinal cortex (BA 35), left middle frontal gyrus (BA 46), left VLPFC (BAs 44 and 47), right precuneus (BA 7), and left middle occipital cortex (BA 19; see Table 3). Thus, vividness may account for some, but not all, of the differences observed between AM and ToM.

\section{DISCUSSION}

The aim of this study was to advance the understanding of the brain regions supporting AM and ToM by directly comparing the two abilities within the same study and against a common baseline, taking into account separate construction and elaboration phases of event generation (Addis et al., 2007; Conway et al., 2001, 2003). Until recently (Spreng \& Grady, in press; Andrews-Hanna et al., 2007), evidence of a shared brain network underlying AM and ToM has been based on comparisons of independent neuroimaging studies investigating one ability or the other (e.g.,
Spreng et al., 2009; Buckner \& Carroll, 2007; Hassabis \& Maguire, 2007). Most of these studies have focused on common brain regions, paying little or no attention to areas of unique activity. However, patient studies have indicated that $\mathrm{AM}$ and ToM are functionally dissociable (Rosenbaum et al., 2007; see also Bird, Castelli, Malik, Frith, \& Hussain, 2004), suggesting that each ability is supported by unique neural correlates. Our data indicate that both common and distinct patterns of activity mediate the remembering and imagining of AM and ToM events, respectively. There are three main findings. First, our analyses largely confirmed areas of activity common to AM and ToM within frontal and posterior/medial temporal lobe regions, but even within this common network, midline structures were engaged to a greater extent during AM. Second, the right hippocampus was uniquely engaged during the construction of AM events, whereas more lateral regions, including left VLPFC (BA 44/45), left precentral gyrus (BA 4), left STG (BA 22), 
and right TPJ (BA 39), were uniquely engaged during the elaboration of ToM events. Third, a region of left perirhinal cortex (BA 35) extending into the left hippocampus was modulated by event vividness based on complementary findings of increased activity in this region during AM, which only included vivid events, and when vivid ToM events were compared to vague ToM events. This suggests that the greater activation observed in left perirhinal cortex might be a function of more vivid event generation during AM relative to ToM.

\section{Regions Commonly Engaged by Vivid AM and ToM Events}

We found a common set of brain regions underlying AM and ToM, including bilateral hippocampus and perirhinal cortex (BAs 35 and 36), bilateral paracingulate cortex (BA 32), bilateral frontal poles (BA 10), a region of left VLPFC (BA 47), bilateral posterior cingulate/retrosplenial cortex (BA 29/ 30), bilateral MTG (BA 21), and right TP (BA 38). Separate analysis of construction and elaboration phases of AM and ToM events indicated that overlapping regions predominated during the elaboration phase. These regions are remarkably similar to those reported in other studies comparing episodic memory or AM and ToM either within the same study (Spreng \& Grady, in press; Andrews-Hanna et al., 2007) or across studies (Spreng et al., 2009; Buckner \& Carroll, 2007; Hassabis \& Maguire, 2007).

The mechanism driving the activation of this core network has been a recent and hotly debated topic (e.g., Schacter et al., 2008; Buckner \& Carroll, 2007; Hassabis \& Maguire, 2007). Several hypotheses have been put forth to account for this overlap, including self-projection (Buckner \& Carroll, 2007), scene construction (Hassabis \& Maguire, 2007), and the constructive episodic-simulation hypothesis (Schacter et al., 2008). Although we did not directly test Buckner and Carroll's (2007) theory of self-projection, participants were instructed to generate novel events for the ToM task, and we excluded ToM events that were based on participants' personal memories, thereby minimizing the aspect of self-projection that relies upon personal re- experiencing similar to Hassabis, Kumaran, and Maguire (2007). This suggests that self-projection as it relates to mental time travel cannot fully account for the shared pattern of activity that we observed. However, this does not exclude the possibility that other, more generic aspects of self-referential processing are at play when generating ToM events.

Our findings fit well with some aspects of the constructive episodic-simulation hypothesis and related ideas, which suggest that episodic memory provides a source of details that can be flexibly recombined to generate coherent, novel events (Schacter et al., 2008; Schacter \& Addis, 2007), and that the hippocampus supports relational processing that involves binding of co-occurring elements of an event (Davachi, 2004; Ryan, Althoff, Whitlow, \& Cohen, 2000; Cohen et al., 1999). Rosenbaum et al. (2009) built on these ideas by suggesting that the hippocampus may play a role in generating and/or binding episodic details, whether real or imagined, so that those details may be held in working memory while a decision is made regarding the need for further details. Regions of PFC may also be involved to ensure that narratives make sense at a global level and to distinguish real from imagined experiences.

Scene construction has been proposed as an alternative mechanism driving shared patterns of activity. However, in the current study, the need to (re)construct spatial (i.e., scene) and other perceptual elements associated with the probed event was likely reduced by giving participants cues that inherently contain rich spatial and visual information (i.e., family photos), unlike cue words, which are typically used (e.g., Addis et al., 2007; Hassabis, Kumaran, \& Maguire, 2007). Bilateral hippocampal activity was, nonetheless, present during both AM and ToM event elaboration. Scoring of the narratives based on the autobiographical interview procedure (Levine et al., 2002) confirmed that place (spatial) details contained within participants' postscan narratives did not prevail in either condition. More specifically, in the AM condition, participants produced significantly more event-specific details compared to temporal, perceptual, and thought/emotion details. There was also a trend toward participants producing significantly more

Figure 2. (A) Sagittal slice of the right hemisphere showing unique right hippocampal activation during AM construction at a threshold of $p<.0005$ (corrected) (for coordinates of the right hippocampus and other regions, see Table 2). The functional maps are overlaid on the average anatomical image from all participants. Activations shown include areas $>120 \mathrm{~mm}^{3}$. (B) Sagittal slice of the left hemisphere showing greater left hippocampal/perirhinal (BA 35) involvement during the generation of vivid ToM events relative to vague ToM events at a threshold of $p<.05$ (corrected) (for coordinates of the left hippocampus/perirhinal cortex and other regions, see Table 3). 
Table 2. Regions Commonly and Differentially Engaged by Vivid AM and ToM Event Construction and Elaboration

\begin{tabular}{|c|c|c|c|c|c|}
\hline \multirow[b]{2}{*}{ Brain Regions } & \multicolumn{3}{|c|}{ Coordinates } & \multirow[b]{2}{*}{$t$} & \multirow[b]{2}{*}{ Vol. } \\
\hline & $x$ & $y$ & $z$ & & \\
\hline \multicolumn{6}{|l|}{$A M$ and ToM Construction $>$ Baseline } \\
\hline \multirow[t]{2}{*}{ R. Entorhinal/perirhinal cortex (BA 28/35) } & 22 & -22 & -10 & 7.07 & 913 \\
\hline & 19 & -25 & -18 & 3.83 & 1794 \\
\hline L. Parahippocampal cortex (BA 36) & -26 & -37 & -17 & 7.07 & 572 \\
\hline L. Paracingulate cortex (BA 32) ${ }^{\mathrm{a}}$ & -3 & 46 & 2 & 5.61 & 198 \\
\hline L. Frontal pole $(\mathrm{BA} 10)^{\mathrm{a}}$ & -4 & 49 & 1 & 5.00 & 357 \\
\hline L. Middle frontal gyrus (BA 8) & -18 & 32 & 50 & 5.61 & 1139 \\
\hline L. Ventrolateral PFC (BA 47) & -39 & 15 & -8 & 3.83 & 1356 \\
\hline L. PCC (BA 31/23) ${ }^{\mathrm{a}}$ & -3 & -55 & 22 & 7.07 & 1755 \\
\hline L. Precuneus (BA 31/23) ${ }^{\mathrm{a}}$ & 0 & -63 & 24 & 7.07 & 255 \\
\hline R. Inferior occipital cortex (BA 18/19) & 39 & -80 & -12 & 7.80 & 185 \\
\hline L. Inferior occipital cortex (BA 19) & -39 & -80 & & 7.80 & 469 \\
\hline \multicolumn{6}{|l|}{$A M$ and ToM Elaboration $>$ Baseline } \\
\hline R. Hippocampus & 30 & -17 & -11 & 5.61 & 1038 \\
\hline L. Hippocampus & -26 & -19 & -13 & 3.83 & 22683 \\
\hline \multirow[t]{2}{*}{ R. Perirhinal cortex (BA 35) } & 21 & & -13 & 3.35 & 2661 \\
\hline & 20 & & -12 & 5.61 & 996 \\
\hline R. Entorhinal cortex (BA 28) & 25 & -21 & -12 & 3.35 & 1819 \\
\hline \multirow[t]{2}{*}{ L. Entorhinal/perirhinal cortex (BA 28/35) } & -22 & -19 & -12 & 3.35 & 1789 \\
\hline & & -18 & -13 & 2.31 & 1536 \\
\hline L. Paracingulate cortex (BA 32) ${ }^{\mathrm{a}}$ & -1 & 45 & 0 & 3.35 & 13154 \\
\hline L. ACC (BA 25) ${ }^{\mathrm{a}}$ & 0 & 12 & 0 & 3.35 & 550 \\
\hline L. Frontal pole (BA 10) & -9 & 54 & -1 & 3.35 & 1386 \\
\hline L. Superior frontal gyrus (BA 8) & -18 & 32 & 51 & 3.35 & 261 \\
\hline L. Dorsomedial PFC (BA 9) & -17 & 32 & 30 & 3.35 & 2154 \\
\hline L. Ventrolateral PFC (BA 47) & -45 & 32 & -3 & 3.35 & 430 \\
\hline L. PCC/RSC (BA 30) & -18 & -57 & 12 & 3.35 & 2209 \\
\hline L. PCC/precuneus (BA 31/23/30) ${ }^{a}$ & -5 & -56 & 21 & 3.35 & 638 \\
\hline L. Middle temporal gyrus (BA 21) & -61 & -4 & -13 & 3.35 & 826 \\
\hline R. Inferior temporal gyrus (BA 20) & 62 & -7 & -21 & 3.35 & 1039 \\
\hline R. Fusiform gyrus (BA 37) & 37 & -43 & -19 & 3.35 & 305 \\
\hline L. Temporal pole (BA 38) & -50 & 13 & -12 & 3.35 & 856 \\
\hline R. Inferior occipital cortex (BA 18/19) & 38 & -81 & -15 & 3.35 & 399 \\
\hline \multicolumn{6}{|l|}{$A M>$ ToM Construction } \\
\hline R. Hippocampus & 32 & -14 & -16 & 7.07 & 647 \\
\hline L. Hippocampus & -29 & -22 & -16 & 7.84 & 162 \\
\hline
\end{tabular}


Table 2. (continued)

\begin{tabular}{|c|c|c|c|c|c|}
\hline \multirow[b]{2}{*}{ Brain Regions } & \multicolumn{3}{|c|}{ Coordinates } & \multirow[b]{2}{*}{$t$} & \multirow[b]{2}{*}{ Vol. } \\
\hline & $x$ & $y$ & $z$ & & \\
\hline R. Entorhinal/perirhinal cortex (BA 28/35)* & 22 & -20 & -11 & 2.31 & 1789 \\
\hline L. Parahippocampal cortex (BA 36/37) & -26 & -43 & -8 & 3.83 & 178 \\
\hline L. Entorhinal cortex (BA 34) & -24 & -1 & -10 & 3.35 & 152 \\
\hline R. Superior frontal gyrus (BA 8) & 21 & 47 & 39 & 3.35 & 189 \\
\hline R. Dorsolateral PFC (BA 9) & 43 & 4 & 24 & 3.35 & 1031 \\
\hline R. Ventrolateral PFC (BA 47) & 31 & 33 & -8 & 5.609 & 262 \\
\hline R. Lingual gyrus (BA 19) & 22 & -61 & -5 & 3.83 & 261 \\
\hline L. Lingual gyrus (BA 18) & -8 & -63 & 4 & 3.35 & 373 \\
\hline
\end{tabular}

ToM > AM Construction

No areas

AM > ToM Elaboration

R. Perirhinal cortex (BA 35)*

L. Perirhinal cortex (BA 36)*

L. Paracingulate cortex (BA 32)

L. Frontal pole (BA 10)

L. Superior frontal gyrus (BA 9/10)

R. Superior frontal gyrus (BA 9)

R. Superior frontal gyrus (BA 46)

L. PCC/RSC (BA 29/30/23) $)^{a}$

L. Precuneus (BA 18/31) ${ }^{a}$

$\begin{array}{rrrrr}19 & -24 & -11 & 2.31 & 264 \\ -26 & -24 & -25 & 2.31 & 296 \\ -6 & 44 & 1 & 3.35 & 171 \\ -6 & 51 & 9 & 3.35 & 360 \\ -17 & 53 & 16 & 3.35 & 263 \\ 4 & 58 & 33 & 3.35 & 398 \\ 46 & 40 & 19 & 3.35 & 294 \\ -1 & -56 & 13 & 3.83 & 1766 \\ -2 & -74 & 28 & 3.83 & 721\end{array}$

TOM > AM Elaboration

L. Ventrolateral PFC (BA 44/45)

L. Precentral gyrus (BA 4)

L. Ventrolateral PFC/STG (BA 44/22)

$\begin{array}{rrr}-52 & 17 \\ -34 & -24 & 5 \\ -57 & 6 & 3 \\ -60 & -58 & 1 \\ 55 & -45 & 3 \\ -59 & -52 & \end{array}$

7

3.35

141

57

3.83

381

L. STG (BA 22)

4

3.83

657

R. Temporo-parietal junction (BA 39)

31

3.83

193

L. Supramarginal gyrus (BA 40)

19

3.83

394

All activations reported survived a threshold of $p<.01$ (corrected). For each cluster of activation, the Talairach coordinates of the maximally activated voxel within each structure are reported, as indicated by the highest $t$ value. AM $=$ autobiographical memory; ToM $=$ theory of mind; Vol. $=$ cluster volume $\left(\mathrm{mm}^{3}\right) ; \mathrm{R}=$ right; $\mathrm{L}=$ left; $\mathrm{BA}=$ Brodmann's area; $\mathrm{PFC}=$ prefrontal cortex; $\mathrm{PCC}=$ posterior cingulate cortex; ACC $=$ anterior cingulate cortex; RSC $=$ retrosplenial cortex; STG $=$ superior temporal gyrus.

In the case where two coordinates are provided due to slight differences in the peak area of activation, the first corresponds to AM and the second to ToM. ${ }^{a}$ Although, the only local maximum for this structure was left lateralized, this activation was bilateral.

*A priori region of interest, $p<.05$.

event-specific details relative to place details. In the ToM condition, participants produced significantly more event-specific details relative to place, temporal, and perceptual details, as well as significantly more emotion/thought details compared to temporal details. It is also possible that some regions within this common network reflect similar demands on semantic processing (Rosenbaum et al., 2009), but it remains for future research to directly test these possibly related accounts. 


\section{Regions of Overlap Are Engaged to a Greater Extent during AM}

We found areas within mPFC common to AM and ToM, yet a direct comparison revealed greater activity within bilateral frontal poles (BA 10) and paracingulate cortex (BA 32) during AM relative to ToM. A similar pattern emerged during event elaboration, although activity was mostly left-lateralized. mPFC regions have been associated with processing information about the self and other people, including the ability to represent another person's perspective during ToM (for a review, see Amodio \& Frith, 2006). There is debate in the literature regarding the fractionation of the MPFC and whether dissociable regions play a specialized role in thinking about the self and reasoning about other people's minds. Saxe et al. (2006), for example, contend that mPFC plays a general role in both types of "mentalizing." By contrast, Mitchell (2006) and Mitchell, Banaji, and MacRae (2005) have found that ventral regions predominate during tasks that involve self-referential processing, whereas more dorsal regions are engaged when inferring the contents of other people's minds, particularly when the other person is dissimilar from oneself. Our conjunction analysis did suggest a trend toward a more ventral pattern of activity for AM and a more dorsal one for ToM (see also Figure 1). Thus, although mPFC likely mediates processes common to AM and ToM, there may be a dissociation between representing the self and representing other people's mental states, in line with Mitchell and colleagues' suggestion.

Parahippocampal cortex exhibited greater activity during AM relative to ToM. This may reflect the recollection of sensory-perceptual and/or spatial contextual details associated with recalling real events compared to imagining novel events in the ToM condition (e.g., Bar, Aminoff, \& Schacter, 2008; Hayes, Nadel, \& Ryan, 2007). During the elaboration phase, greater activity was observed during AM relative to ToM within bilateral posterior cingulate/ retrosplenial cortex (BA 29/30/23) and precuneus (BA 18/ 31). These regions are consistently activated in studies of AM (Cabeza \& St. Jacques, 2007; Svoboda, McKinnon, \& Levine, 2006; Gilboa et al., 2004; Fletcher et al., 1995) and may relate to the vividness or familiarity of an AM during visual imagery of the event (Gilboa et al., 2004).

Our findings of greater activity of bilateral posterior cingulate cortex, bilateral parahippocampal gyrus, and bilateral precuneus during AM elaboration relative to ToM elaboration are also consistent with those of Szpunar et al. (2007), who observed greater activity in these regions when imagining past and future events relative to imagining events involving Bill Clinton (which may involve ToM). Taken together, the two studies suggest that these regions may respond more strongly to familiar events involving the self and possibly when the self is projected across time.

It is unclear, however, if any of the differences observed between AM and ToM relate to processes involved in viewing one's own face in the AM, but not ToM, photos. Although the literature suggests a right hemisphere dominance in self-face recognition, there is a lack of convergence as to the precise neural substrates (Keenan, Wheeler, Gallup, \& Pascual-Leone, 2000).

\section{Areas of Activation Unique to AM and to ToM}

Although the bilateral hippocampus was activated to a similar extent during AM and ToM event elaboration, right hippocampal activity emerged as unique to the construction

Table 3. Regions Differentially Engaged by Vivid and Vague ToM Events

\begin{tabular}{|c|c|c|c|c|c|}
\hline \multirow[b]{2}{*}{ Brain Regions } & \multicolumn{3}{|c|}{ Coordinates } & \multirow[b]{2}{*}{$t$} & \multirow[b]{2}{*}{ Vol. } \\
\hline & $x$ & $y$ & $z$ & & \\
\hline \multicolumn{6}{|l|}{ Vivid ToM > Vague ToM } \\
\hline L. Hippocampus/perirhinal cortex (BA 35)* & -23 & -13 & -25 & 2.12 & 409 \\
\hline R. Perirhinal cortex (BA 35)* & 22 & -21 & -17 & 2.12 & 211 \\
\hline L. Middle frontal gyrus (BA 46) & -43 & 32 & 19 & 2.92 & 316 \\
\hline L. Ventrolateral PFC (BA 44) & -50 & 7 & 22 & 2.92 & 395 \\
\hline L. Ventrolateral PFC (BA 47) & -46 & 30 & 1 & 2.92 & 226 \\
\hline R. Precuneus (BA 7) & 21 & -67 & 40 & 2.92 & 151 \\
\hline L. Fusiform gyrus (BA 37) & -51 & -51 & -15 & 2.92 & 335 \\
\hline L. Middle occipital cortex (BA 19) & -38 & -83 & 18 & 2.92 & 151 \\
\hline
\end{tabular}

All activations reported survived a threshold of $p<.01$ (corrected). For each cluster of activation, the Talairach coordinates of the maximally activated voxel within each structure are reported, as indicated by the highest $t$ value. ToM $=$ theory of mind; Vol. $=$ cluster volume $\left(\mathrm{mm}^{3}\right) ; \mathrm{L}=$ left; $\mathrm{R}=$ right; $\mathrm{BA}=$ Brodmann's area; $\mathrm{PFC}=$ prefrontal cortex

*A priori region of interest, $p<.05$. 
phase of AM, suggesting that this region may support functions that are specific to the retrieval of real events involving the self (Summerfield, Hassabis, \& Maguire, 2009). In the current study, we used personalized cues to probe past memories, which might evoke the recollection of a past personal event via hippocampally mediated memory traces (Moscovitch, 1992; Tulving, 1983). Previous studies using impersonal cues, such as generic cue words or nouns, did not result in right hippocampal activation during the construction of AM events (e.g., Addis et al., 2007; Conway et al., 2003). However, a recent fMRI study that cued participants with sentences of personal events that were collected during a prescan interview resulted in right hippocampal activation 6 to 8 sec after stimulus onset (Addis, Pan, $\mathrm{Vu}$, Laiser, \& Schacter, in press). Although we found activation in this region within the first 4 sec of stimulus onset, it is possible that this difference is due to the use of personal photos, which serve as highly specific retrieval cues and, therefore, reduce the time needed to search and retrieve specific memories.

Another possibility is that activity in this region may be driven by the initial spatial or relational processing of complex visual scenes within the photos at encoding (e.g., Hassabis \& Maguire, 2007; Binder, Bellgowan, Hammeke, Possing, \& Frost, 2005; Köhler, Crane, \& Milner, 2002). It is notable that hippocampal recruitment was not apparent during the construction phase of the ToM condition, arguing against perceptual discrimination of scenes or other objects depicted in the photos as accounting for this region's involvement in the present case (e.g., Lee, Scahill, \& Graham, 2008; Lee, Bandelow, Schwarzbauer, Henson, \& Graham, 2006). Moreover, it is unlikely that right hippocampal activity is due to vividness, as this region did not emerge in the comparison of vivid versus vague ToM events.

Bilateral activity of the dorsal medial nucleus of the thalamus was also unique to AM when events were collapsed across construction and elaboration phases. This is consistent with findings from patient and neuroimaging studies (e.g., Svoboda et al., 2006; Miller et al., 2001; McCarthy \& Hodges, 1995). Miller, Caine, and Watson (2003) and Miller et al. (2001) suggest that the thalamus may be needed to recall unique or highly specific details, whether episodic or semantic, but not for accessing more general information. Similarly, evidence from neuroimaging research suggests that thalamic activity is associated with the generation of specific (exemplar) episodic autobiographical images (Gardini, Cornoldi, De Beni, \& Venneri, 2006; Gardini, De Beni, Cornoldi, Bromiley, \& Venneri, 2005). It may be that in healthy individuals, the generation of AMs is more likely to induce the retrieval of eventspecific details, whereas ToM draws on generic or schematic representations. This might also be signaled by the recruitment of left VLPFC (BA 44/45) during ToM but not $\mathrm{AM}$, as this region is involved in tasks requiring access to semantic information (Devlin, Matthews, \& Rushworth, 2003; Poldrack et al., 1999). Activity within left VLPFC (BA 44/45) has emerged in other ToM studies as well.
For example, in a quantitative meta-analysis of the neuroimaging literature, a similar region of left VLPFC $(-50,16,4)$ was uniquely activated in studies of ToM (relative to AM; Spreng et al., 2009). Furthermore, patient work indicates that this region is necessary for mediating emotional empathy, which assumes ToM processes (Shamay-Tsoory, Aharon-Peretz, \& Perry, 2009).

Along with left VLPFC (BA 44/45), we found a number of other lateral regions to be uniquely engaged during ToM elaboration, including the left precentral gyrus (BA 4), left STG (BA 22), and right TPJ. The TPJ, in particular, has been identified as a key region for ToM in patient studies (Samson et al., 2004, 2007; but see Channon et al., 2007) and neuroimaging studies (Lombardo et al., in press; Andrews-Hanna et al., 2007; Gobbini, Koralek, Montgomery, \& Haxby, 2007; Saxe et al., 2006; Saxe \& Kanwisher, 2003). Saxe et al. (2006) and Saxe and Wexler (2005) argue that the right TPJ is selective to the attribution of other people's mental states and is not involved in processing other socially relevant information about a person or one's own mental states.

A recent study using repetitive transcranial stimulation provided additional support for the right TPJ selectivity to ToM (Costa, Torriero, Oliveri, \& Caltagirone, 2008), although patient work suggests that the left TPJ is necessary for performance on a false belief test of ToM (Samson et al., 2004). Further research is needed to determine laterality effects, and whether various ToM tasks are affected differentially by TPJ lesions (see Channon et al., 2007; Gobbini et al., 2007). Involvement of the TPJ, whether right or left, along with areas of the precentral gyrus and lateral temporal cortex, may reflect the role of this network in action-based representations and gaze perception (e.g., Martin, 2007; Pelphrey, Morris, \& McCarthy, 2005; Hooker et al., 2003; Allison, Puce, \& McCarthy, 2000; but see Bedny, Caramazza, Grossman, Pascual-Leone, \& Saxe, 2008).

It is also difficult to disregard the association of the TPJ and surrounding areas of ventral parietal cortex with bottom-up attentional processes (Mitchell, 2008; Corbetta, Kincade, Ollinger, McAvoy, \& Shulman, 2000). Indeed, the involvement of the TPJ in ToM, but not AM, may reflect greater deployment of attention to the former or indicate that ToM serves as a stronger reorienting cue (see also Cabeza et al., 2008). In any case, these findings suggest that several lateral brain regions may support ToM and may explain why we observe normal ToM in individuals with severe AM impairment, but preserved semantic memory, in relation to damage to the hippocampus and/or hippocampal disconnection from frontal regions (Rosenbaum et al., 2007).

Our overall findings of unique activity of lateral frontal and temporal-parietal regions during ToM, together with greater activity of midline regions during AM relative to ToM, corresponds well with the findings reported in a separate neuroimaging study that also directly compared AM to ToM (Spreng \& Grady, in press). The robustness of these findings is highlighted by the differences between 
these two studies; Spreng and Grady employed a functional connectivity analysis, a younger sample, and different stimuli (i.e., nonpersonal photos from the IAPS).

\section{Left Hippocampus/Perirhinal Cortex Modulates Event Vividness}

A region of left perirhinal cortex (BA 35) extending into the left hippocampus exhibited greater activity when vivid ToM events were compared to vague/unclear ToM events, and also during the elaboration of vivid AM and vivid ToM events, although to a greater extent during AM. The greater activity observed during AM may reflect more vivid event generation during the recollection of past personal events relative to imagined events involving other people, which is consistent with participants' postscan vividness ratings. Although coherence per se was not directly examined, it remains viable that the main contribution of the left hippocampus is to facilitate the general coherence of an episode or scene, given that some participants described nonvivid events as fragmented (Hassabis \& Maguire, 2007). In any case, a positive correlation between left hippocampal activity and vividness or amount of detail has been reported in a study using a similar paradigm to examine AM (Gilboa et al., 2004) and in one using cue words to examine future imagining (Addis \& Schacter, 2008). The current study extends this role of the left hippocampus to vivid imagining of nonpersonal events and suggests that the increased likelihood of finding greater left hippocampal activity in studies of AM relative to ToM (Spreng et al., 2009) may be due to the highly vivid nature of re-experiencing personal events in memory. Note that it was not possible to predict the number of ToM events that could be included in the vivid versus vague analysis, as these events were classified according to participants' ratings. Therefore, in some cases, participants contributed very few events to this analysis.

How can neuroimaging evidence of bilateral hippocampal involvement in both AM and ToM be reconciled with existing patient data showing that this region is necessary for AM but not for ToM (Rosenbaum et al., 2007)? This discrepancy emphasizes that similar levels of activation in neuroimaging studies do not predict the necessity of a region for a particular function. Nonetheless, closer inspection of the present data indicates that the hippocampus is not involved in the initial construction phase of ToM and is only jointly involved in AM and ToM when event details are elaborated. Therefore, it is possible that less detailed, and possibly generic, AM and ToM representations may continue to be supported in the face of hippocampal compromise. This is in line with evidence from neuroimaging (Addis, Moscovitch, Crawley, \& McAndrews, 2004; Gilboa et al., 2004) and patient studies (Gilboa et al., 2006). Indeed, ToM of the type that is assessed on standard measures and typical of everyday life is likely to be based on schemas or generic information that is already bound together and does not require elaboration. In rarer instances when ToM requires vivid representations and/or elaboration of event details, individuals with hippocampal amnesia should show impairment, as has been found for imagining of personal experiences (Rosenbaum et al., 2005, 2009; Klein \& Loftus, 2002).

A limitation in the current study was that it was not possible to tease apart differences relating to recalling real events and imagining novel events inherent in AM and ToM, respectively. Therefore, some of the differences we observed may relate to differences between remembering and imagining, and not necessarily to AM and ToM. It may be useful for future studies to include a condition that involves imagining past events that have not yet occurred in order to disentangle the neural correlates of AM and ToM over and above those relating to remembering and imagining. This type of condition has been employed in a recent study comparing AM and imagining oneself in future events (Addis et al., in press).

\section{Conclusion}

We found common and distinct brain regions support $\mathrm{AM}$ and ToM event when compared in the same study using a closely matched paradigm. Bilateral hippocampus, along with other medial frontal, temporal, and parietal regions, were commonly engaged during $\mathrm{AM}$ and ToM, thereby recreating the core network of brain regions supporting the two abilities. Further comparison of AM and ToM events during construction and elaboration phases revealed key differences between the two abilities, including unique right hippocampal activity during AM construction and unique activity of more lateral frontal and temporal-parietal structures during ToM elaboration. The latter finding suggests that the right TPJ and other lateral regions, but not the hippocampus, may be needed for ToM, given that this ability is intact in amnesic people. Additional analyses indicated that a region of the left hippocampus/perirhinal cortex was modulated by event vividness, whether the events are real or imagined, and whether they involve the self or other people. Taken together, these findings highlight important differences between the two abilities and demonstrate the need to investigate AM and ToM against a common baseline within the same study, taking into account the time course of event generation.

\section{Acknowledgments}

This work was funded by a Canadian Institutes of Health Research New Investigator Award and Operating Grant (MOP 93535) to R. S. R. and a Heart and Stroke Foundation of Ontario Master's Studentship Award to J. S. R. We thank the Heart and Stroke Foundation of Ontario Centre for Stroke Recovery and the Louis and Leah Posluns Centre for Stroke and Cognition at Baycrest for their support. A. G. is supported by a FP6 Marie-Curie IRG grant (EU), and D. T. S. is supported by the Reva James Leeds Chair in Neuroscience and Research Leadership. We also thank N. Carson, S. Gillingham, and R. Tong for technical assistance and R. Cabeza, G. Chen, and N. Spreng for useful advice. The research reported 
in this article was completed in partial fulfillment of requirements for J. S. R.'s Master's thesis at York University.

Reprint requests should be sent to Jennifer S. Rabin or R. Shayna Rosenbaum, Department of Psychology, York University, 4700 Keele St., Toronto, ON, Canada M3J 1P3, or via e-mail: shaynar@yorku.ca.

\section{Notes}

1. The term "generated" will be used to collectively refer to the process of recollecting AM events and imagining ToM events. 2. Unlike the other analyses, a within-subjects analysis was not possible, as some participants rated all ToM events as vividly imagined. Given that participants rated significantly more ToM events as vividly imagined than vaguely imagined, we included only a subset of participants in this analysis in order to closely match the total number of vivid and vague ToM events.

3. Postscan narratives from 4 of the 18 participants were excluded from this analysis due to a recording error.

4. Additional details of the results from the postscan narrative analysis are available upon request.

\section{REFERENCES}

Addis, D. R., Moscovitch, M., Crawley, A. P., \& McAndrews, M. P. (2004). Recollective qualities modulate hippocampal activation during autobiographical memory retrieval. Hippocampus. 14, 752-762.

Addis, D. R., Pan, L., Vu, M. A., Laiser, N., \& Schacter, D. L. (in press). Constructive episodic simulation of the future and the past: Distinct subsystems of a core brain network mediate imagining and remembering. Neuropsychologia.

Addis, D. R., \& Schacter, D. L. (2008). Constructive episodic simulation: Temporal distance and detail of past and future events modulate hippocampal engagement. Hippocampus, 18, 227-237.

Addis, D. R., Wong, A. T., \& Schacter, D. L. (2007). Remembering the past and imagining the future: Common and distinct neural substrates during event construction and elaboration. Neuropsychologia. 45, 1363-1377.

Allison, T., Puce, A., \& McCarthy, G. (2000). Social perception from visual cues: Role of the STS region. Trends in Cognitive Sciences, 4, 267-278.

Amodio, D. M., \& Frith, C. D. (2006). Meeting of minds: The medial frontal cortex and social cognition. Nature Reviews: Neuroscience, 7, 268-277.

Andrews-Hanna, J. R., Saxe, R., Poulin, R., \& Buckner, R. L. (2007). The default system overlaps activation during theory of mind and episodic memory retrieval tasks. Society for Neuroscience Abstracts.

Bar, M., Aminoff, E., \& Schacter, D. L. (2008). Scenes unseen: The parahippocampal cortex intrinsically subserves contextual associations, not scenes or places per se. Iournal of Neuroscience, 28, 8539-8544.

Bedny, M., Caramazza, A., Grossman, E., Pascual-Leone, A., \& Saxe, R. (2008). Concepts are more than precepts: The case of action verbs. Journal of Neuroscience, 28, 11347-11353.

Binder, J. R., Bellgowan, P. S. F., Hammeke, T. A., Possing, E. T., \& Frost, J. A. (2005). A comparison of two fMRI protocols for eliciting hippocampal activation. Etilepsia, 46, 1061-1070.

Bird, C. M., Castelli, F., Malik, O., Frith, U., \& Hussain, M. (2004). The impact of extensive medial frontal lobe damage on "theory of mind" and cognition. Brain, 127, 914-928.

Buckner, R. L., Andrews-Hanna, J. R., \& Schacter, D. L. (2008). The brain's default network: Anatomy, function and relevance to disease. Annals of the New York Academy of Sciences, 1124, 1-38.

Buckner, R. L., \& Carroll, D. C. (2007). Self-projection and the brain. Trends in Cognitive Sciences, 11, 49-57.

Cabeza, R., Ciaramelli, E., Olson, I. R., \& Moscovitch, M. (2008). The parietal cortex and episodic memory: An attentional account. Nature Reviews: Neuroscience, 9, 613-625.

Cabeza, R., \& St. Jacques, P. L. (2007). Functional neuroimaging of autobiographical memory. Trends in Cognitive Sciences, 11, 219-227.

Channon, S., Rule, A., Maudgil, D., Martinos, M., Pellijeff, A., Frankl, J., et al. (2007). Interpretation of mentalistic actions and sarcastic remarks: Effects of frontal and posterior lesions on mentalising. Neuropsychologia, 45, 1725-1734.

Cohen, N. J., Ryan, J. D., Hunt, C., Romine, L., Wszalek, T., \& Nash, C. (1999). Hippocampal system and declarative (relational) memory: Summarizing the data from functional neuroimaging studies. Hippocampus, 9, 83-98.

Conway, M. A., Pleydell-Pearce, C. W., \& Whitecross, S. E. (2001). The neuroanatomy of autobiographical memory: A slow cortical potential study of autobiographical memory retrieval. Journal of Memorv and Language, 45, 493-524.

Conway, M. A., Pleydell-Pearce, C. W., Whitecross, S. E., \& Sharpe, H. (2003). Neurophysiological correlates of memory for experienced and imagined events. Neuropsychologia. 41, 334-340.

Corbetta, M., Kincade, J. M., Ollinger, J. M., McAvoy, M. P., \& Shulman, G. L. (2000). Voluntary orienting is dissociated from target detection in human posterior parietal cortex. Nature Neuroscience, 3, 292-297.

Corbetta, M., Patel, G., \& Shulman, G. L. (2008). The reorienting system of the human brain: From environment to theory of mind. Neuron, 58, 306-324.

Corcoran, R., \& Frith, C. D. (2003). Autobiographical memory and theory of mind: Evidence of a relationship in schizophrenia. Psvchological Medicine, 33, 897-905.

Costa, A., Torriero, S., Oliveri, M., \& Caltagirone, C. (2008). Prefrontal and temporo-parietal involvement in taking others' perspective: TMS evidence. Behavioural Neurology, 19, 71-74.

Cox, R. W. (1996). AFNI: Software for analysis and visualization of functional magnetic resonance neuroimages. Computers and Biomedical Research, 29, 162-173.

Davachi, L. (2004). The ensemble that plays together, stays together. Hippocampus, 14, 1-3.

Devlin, J. T., Matthews, P. M., \& Rushworth, M. F. (2003). Semantic processing in the left inferior prefrontal cortex: A combined functional magnetic resonance imaging and transcranial magnetic stimulation study. Journal of Cognitive Neuroscience, 15, 71-84.

Fletcher, P. C., Frith, C. D., Baker, S. C., Shallice, T., Frackowiak, R. S., \& Dolan, R. J. (1995). The mind's eye-Precuneus activation in memory-related imagery. Neuroimage, 2, 195-200.

Frith, U., \& Frith, C. D. (2003). Development and neurophysiology of mentalizing. Philosophical Transactions of the Royal Society of London: Series B, Biological Sciences. 358, 459-473.

Gallagher, H. L., \& Frith, C. D. (2003). Functional imaging of "theory of mind." Trends in Cognitive Sciences, 7, 77-83. Gardiner, J. M., Ramponi, C., \& Richardson-Klavehn, A. (1998). Experiences of remembering, knowing, and guessing. Consciousness \& Cognition, 7, 1-26.

Gardini, S., Cornoldi, C., De Beni, R., \& Venneri, A. (2006). Left mediotemporal structures mediate the retrieval of episodic autobiographical mental images. Neuroimage. 30, 645-655. 
Gardini, S., De Beni, R., Cornoldi, C., Bromiley, A., \& Venneri, A. (2005). Different neuronal pathways support the generation of general and specific mental images. Neuroimage, 27, $544-552$.

Gilboa, A. (2004). Autobiographical and episodic memoryOne and the same? Evidence from prefrontal activation in neuroimaging studies. Neuropsychologia, 42, 1336-1349.

Gilboa, A., Winocur, G., Grady, C. L., Hevenor, S. J., \& Moscovitch, M. (2004). Remembering our past: Functional neuroanatomy of recollection of recent and very remote personal events. Cerebral Cortex, 14, 1214-1225.

Gilboa, A., Winocur, G., Rosenbaum, R. S., Poreh, A., Gao, F., Black, S. E., et al. (2006). Hippocampal contributions to recollection in retrograde and anterograde amnesia. Hippocampus, 16, 966-980.

Gobbini, M. I., Koralek, A. C., Montgomery, K. J., \& Haxby, J. V. (2007). Two takes on the social brain: A comparison of theory of mind tasks. Journal of Cognitive Neuroscience, 19, 1803-1814.

Hassabis, D., Kumaran, D., \& Maguire, E. A. (2007). Using imagination to understand the neural basis of episodic memory. Journal of Neuroscience, 27, 14365-14374.

Hassabis, D., Kumaran, D., Vann, S. D., \& Maguire, E. A. (2007). Patients with hippocampal amnesia cannot imagine new experiences. Proceedings of the National Academy of Sciences, U.S.A., 104, 1726-1731.

Hassabis, D., \& Maguire, E. A. (2007). Deconstructing episodic memory with construction. Trends in Cognitive Sciences, 11, 299-306.

Hayes, S. M., Nadel, L., \& Ryan, L. (2007). The effect of scene context on episodic object recognition: Parahippocampal cortex mediates memory encoding and retrieval success. Hippocampus, 17, 873-889.

Hooker, C. L., Paller, K. A., Gitelman, D. R., Parrish, T. B., Mesulam, M. M., \& Reber, P. J. (2003). Brain networks for analyzing eye gaze. Cognitive Brain Research, 17, 406-418.

Keenan, J. P., Wheeler, M. A., Gallup, G. G., Jr., \& Pascual-Leone, A. (2000). Self-recognition and the right prefrontal cortex. Trends in Cognitive Sciences, 4, 338-344.

Klein, S. B., \& Loftus, J. (2002). Memory and temporal experience: The effects of episodic memory loss on an amnesic patient's ability to remember the past and imagine the future. Social Cognition, 20, 353-379.

Köhler, S., Crane, J., \& Milner, B. (2002). Differential contributions of the parahippocampal place area and the anterior hippocampus to human memory for scenes. Hippocampus, 12, 718-723.

Lang, P. J., Bradley, M. M., \& Cuthbert, B. N. (2005). International affective picture system (IAPS): Affective ratings of pictures and instruction manual. Technical Report A-6. Gainesville, FL: University of Florida.

Lee, A. C. H., Bandelow, S., Schwarzbauer, C., Henson, R. N. A., \& Graham, K. S. (2006). Perirhinal cortex activity during visual object discrimination: An event-related fMRI study. Neuroimage, 33, 362-373.

Lee, A. C. H., Scahill, V. L., \& Graham, K. S. (2008). Activating the medial temporal lobe during oddity judgment for faces and scenes. Cerebral Cortex, 18, 683-696.

Levine, B., Svoboda, E., Hay, J. F., Winocur, G., \& Moscovitch, M. (2002). Aging and autobiographical memory: Dissociating episodic from semantic retrieval. Psychology and Aging, 17, 677-689.

Lombardo, M. V., Chakrabarti, B., Bullmore, E. T., Wheelwright, S. J., Sadek, S. A., Suckling, J., et al. (in press). Shared neural circuits for mentalizing about the self and others. Journal of Cognitive Neuroscience.

Martin, A. (2007). The representation of object concepts in the brain. Annual Review of Psychology, 58, 25-45.
McCarthy, R. A., \& Hodges, J. R. (1995). Trapped in time: Profound autobiographical memory loss following a thalamic stroke. In R. Campbell \& M. A. Conway (Eds.), Broken memories: Case studies in memory impairment (pp. 31-44). Cambridge, MA: Blackwell.

Miller, L. A., Caine, D., Harding, A., Thompson, E. J., Large, M., \& Watson, J. D. G. (2001). Right medial thalamic lesion causes isolated retrograde amnesia. Neuropsychologia, 39, 1037-1046.

Miller, L. A., Caine, D., \& Watson, J. D. G. (2003). A role for the thalamus in memory for unique entities. Neurocase, 9 , 504-514.

Mitchell, J. P. (2006). Dissociable medial prefrontal contributions to judgments of similar and dissimilar others. Neuron, 50, 655-663.

Mitchell, J. P. (2008). Activity in right temporo-parietal junction is not selective for theory of mind. Cerebral Cortex, 18, 262-271.

Mitchell, J. P., Banaji, M. R., \& MacRae, C. N. (2005). The link between social cognition and self referential thought in the medial prefrontal cortex. Journal of Cognitive Neuroscience, 17, 1306-1315.

Moscovitch, M. (1992). Memory and working-with-memory: A component process model based on modules and central systems. Journal of Cognitive Neuroscience, 4, 257-267.

O'Keefe, J., \& Nadel, L. (1978). The hippocampus as a cognitive map. Oxford, UK: Clarendon Press.

Okuda, J., Fujii, T., Ohtake, H., Tsukiura, T., Tanji, K., Suzuki, K., et al. (2003). Thinking of the future and past: The roles of the frontal pole and the medial temporal lobes. Neuroimage, 19, 1369-1380.

Pelphrey, K. A., Morris, J. P., \& McCarthy, G. (2005). Neural basis of eye gaze processing deficits in autism. Brain, 128, 1038-1048.

Poldrack, R. A., Wagner, A. D., Prull, M. W., Desmond, J. E., Glover, G. H., \& Gabrieli, J. D. E. (1999). Functional specialization for semantic and phonological processing in the left inferior prefrontal cortex. Neuroimage, 10, 15-35.

Premack, D. G., \& Woodruff, G. (1978). Does the chimpanzee have a theory of mind? Behavioral and Brain Sciences, $1,515-526$.

Rosenbaum, R. S., Gilboa, A., Levine, B., Winocur, G., \& Moscovitch, M. (2009). Amnesia as impairment of detail generation and binding: Evidence from personal, fictional, and semantic narratives in K. C. Neuropsychologia, 47, $2181-2187$.

Rosenbaum, R. S., Köhler, S., Schacter, D. L., Moscovitch, M., Westmacott, R., Black, S. E., et al. (2005). The case of K. C.: Contributions of a memory-impaired person to memory theory. Neuropsychologia, 43, 989-1021.

Rosenbaum, R. S., Moscovitch, M., Foster, J. K., Schnyer, D. M., Gao, F., Kovacevic, N., et al. (2008). Patterns of autobiographical memory loss in medial temporal lobe amnesic patients. Journal of Cognitive Neuroscience, 20, 1490-1506.

Rosenbaum, R. S., Stuss, D. T., Levine, B., \& Tulving, E. (2007). Theory of mind is independent of episodic memory. Science, 318, 1257.

Ryan, J. D., Althoff, R. R., Whitlow, S., \& Cohen, N. J. (2000). Amnesia is a deficit in relational memory. Psychological Science, 11, 454-461.

Samson, D., Apperly, I. A., Chiavarino, C., \& Humphreys, G. W. (2004). The left temporo-parietal junction is necessary for representing someone else's belief. Nature Neuroscience, 7, 499-500.

Saxe, R., \& Kanwisher, N. (2003). People thinking about thinking people: The role of the temporo-parietal junction in "theory of mind." Neuroimage, 19, 1835-1842. 
Saxe, R., Moran, J. R., Scholz, J., \& Gabrieli, J. D. E. (2006). Overlapping and non-overlapping brain regions for theory of mind and self-reflection in individual subjects. Social Cognitive and Affective Neuroscience, 1, 229-234.

Saxe, R., \& Wexler, A. (2005). Making sense of another mind: The role of the right temporo-parietal junction. Neuropsychologia, 43, 1391-1399.

Schacter, D. L., \& Addis, D. R. (2007). The cognitive neuroscience of constructive memory: Remembering the past and imagining the future. Philosophical Transactions of the Royal Society of London: Series B, Biological Sciences, 362, 773-786.

Schacter, D. L., Addis, D. R., \& Buckner, R. L. (2008). Episodic simulation of future events: Concepts, data and applications. Annals of the New York Academy of Sciences, 1124, 39-60.

Shamay-Tsoory, S. G., Aharon-Peretz, J., \& Perry, D. (2009). Two systems for empathy: A double dissociation between emotional and cognitive empathy in inferior frontal gyrus versus ventromedial prefrontal lesions. Brain, 132, 617-627.

Sheldon, M. R., Fillyaw, M. J., \& Thompson, W. D. (1996). The use and interpretation of the Friedman test in the analysis of ordinal-scale data in repeated measures designs. Physiotherapy Research International, 1, 221-228.

Shrout, P. E., \& Fleiss, J. L. (1979). Intraclass correlations: Uses in assessing reliability. Psychological Bulletin, 86, 420-428.
Spreng, R. N., \& Grady, C. L. (in press). Patterns of brain activity supporting autobiographical memory, prospection and theory-of-mind and their relationship to the default mode network. Journal of Cognitive Neuroscience.

Spreng, R. N., Mar, R. A., \& Kim, A. S. (2009). The common neural basis of autobiographical memory, prospection, navigation, theory of mind and the default mode: A quantitative meta-analysis. Journal of Cognitive Neuroscience, 21, 489-510.

Summerfield, J. J., Hassabis, D., \& Maguire, E. A. (2009). Cortical midline involvement in autobiographical memory. Neuroimage, 44, 1188-1200.

Svoboda, E., McKinnon, M. C., \& Levine, B. (2006). The functional neuroanatomy of autobiographical memory: A meta-analysis. Neuropsychologia, 44, 2189-2208.

Szpunar, K. K., Watson, J. M., \& McDermott, K. B. (2007). Neural substrates of envisioning the future. Proceedings of the National Academy of Sciences, U.S.A., 104, 642-647.

Talairach, J., \& Tournoux, P. (1988). Co-planar stereotaxic atlas of the buman brain. New York: Thieme.

Tulving, E. (1983). Elements of episodic memory. Oxford, UK: Clarendon Press.

Tulving, E. (1985). Memory and consciousness. Canadian Psychology, 26, 1-12.

Wheeler, M. A., Stuss, D. T., \& Tulving, E. (1997). Toward a theory of episodic memory: The frontal lobes and autonoetic consciousness. Psychological Bulletin, 121, $331-354$ 